\title{
ENERGETIC IMPACT OF PREHEATING OF FRESH AIR IN ADDED CONVEX WINDOW
}

\author{
ATTILA KEREKES \\ University of Debrecen, Faculty of Engineering, Department of Building Services and Building Engineering, \\ 4028 Debrecen, Ótemető u. 2-4, Hungary \\ E-mail: akerekesa@gmail.com
}

\begin{abstract}
During the renovation of an existing building keeping the original window and installing an added convex window is beneficial for the reduction of heating energy demand, furthermore this solution is free of the disposal and/or recycling of demolition waste. The energy need for heating can be further reduced, if the natural ventilation of the premise is through the buffer zone of the added convex window. In this case the fresh air is pre-heated in the buffer zone, increasing the utilised passive solar gain of the building. Measurements were carried out on experimental huts for analysis of this impact. Dynamic simulations of the huts have been carried out as well. In this paper the results will be presented, which proves the energetic effect.
\end{abstract}

Keywords: added convex window, fresh air pre-heating, experimental huts, measurement, dynamic simulation

\section{Introduction}

The added convex window and its impact on the energy need for heating of the buildings have already been described in several articles [1-3]. The results of the numerous detailed dynamic simulations showed, that in case of given original wall and window structures and a specific geographical place the preheated fresh air operation mode results in the lowest energy need for heating. In this operational mode the supplied air is channelled through the buffer zone of the added convex window, where the fresh air will be pre-heated before entering the room. The energy need of the preheating is covered partly by the green house effect occurring in the buffer zone, partly by the transmitted heat loss of the original window (Fig. 1).

For presenting the energy effect of the added convex window measurements were carried out.

\section{Materials and calculation methods}

The aim of in situ measurements was the following:

1) To prove the energetic effect of the added convex window.

2) To compare the results of the measurements and the simulations in order to validate the last.
For meeting the criterion of the 1st point, measurements were carried out in two identical experimental huts, at the same time, with the only one difference: with added convex window in one of them whilst with traditional window in the other. The identical parameters of the huts matched, such as sizes, used materials and their material characteristics, the building technology, the operation mode, the mode of ventilation and the air change rate, the heating system, the heating control system, values of the set-point parameter, the mode of placement and the location (the locations had to be close to each other), the orientation, therefore the meteorological relations.

The experimental huts could be rotated thus measurements have been repeated with different orientations of the window.

Based on strictly the same conditions of the parameters and the measurement's circumstances, the difference between the measured results is only caused by one thing, the added convex window itself (being the only difference between the two huts), therefore thanks to this method, the energetic effect of the added convex window can be reliably shown.

Meeting the criterion of the 2nd point, simulation has been carried out with the real data of the location of the experiment.

Open Acces statement. This is an open-access article distributed under the terms of the Creative Commons Attribution 4.0 International License (https://creativecommons.org/licenses/by/4.0/), which permits unrestricted use, distribution, and reproduction in any medium, provided the original author and source are credited, a link to the CC License is provided, and changes - if any - are indicated. (SID_1) 


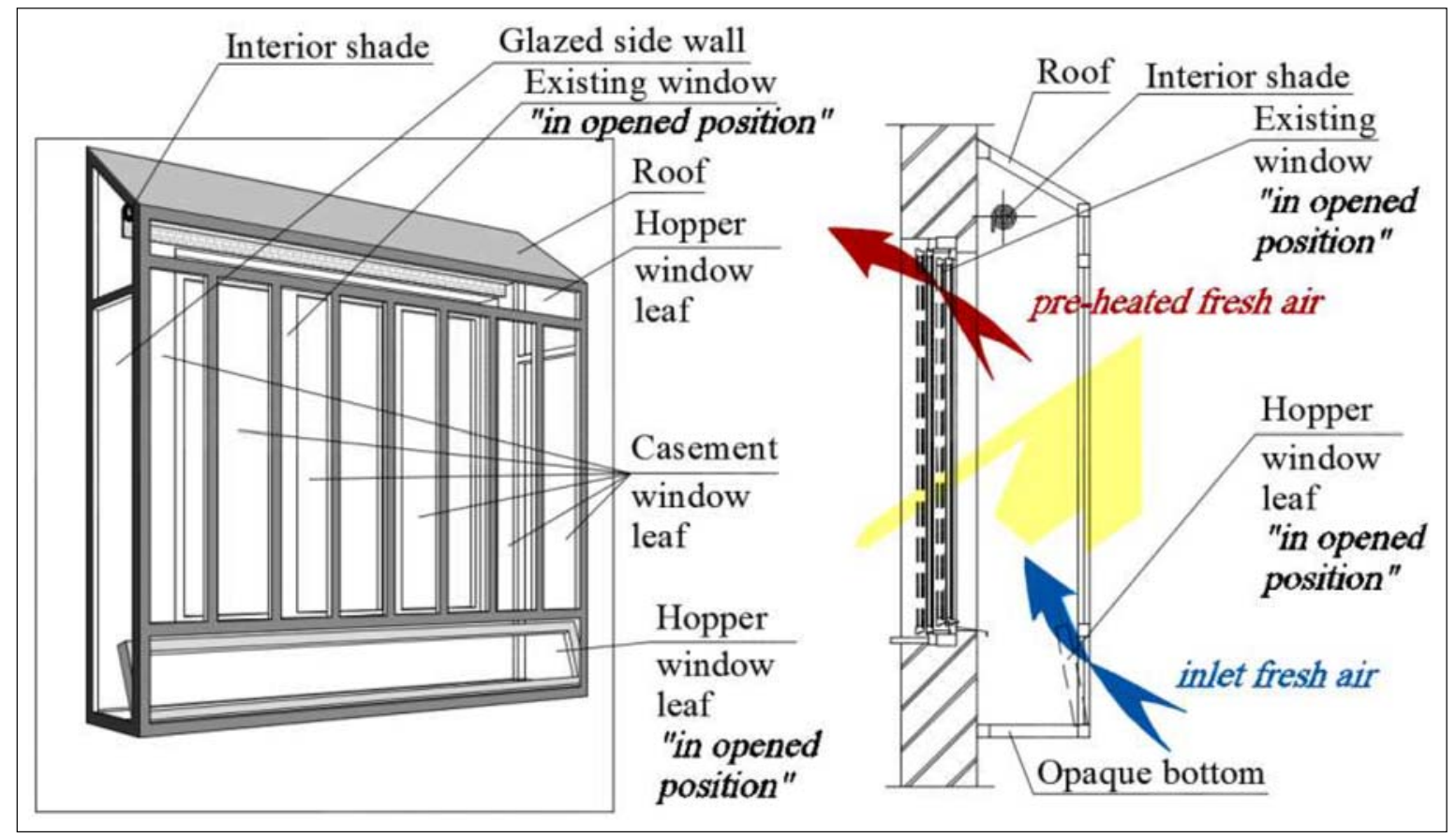

Fig. 1. Sketch of added convex window operating in fresh air preheating mode

For matching appropriately all the criteria, for measuring the summer overheating of the added convex window [4], the modifications of the huts needed to be done. The huts were placed in the same place, with the same orientation. In the heating season in the same operation conditions the data of the inner temperature and the energy consumption were logged. It was possible to change the operation and the operation mode according to the demand. The place of the meas- urement was the Agrometeorological Observatory of the AKIT DTTI Agrometeorological and Agro-ecological Monitoring Centre, University of Debrecen (it is located in Debrecen-Kismacs, the outskirts of Debrecen, $2 \mathrm{~km}$ North-west of the city at Lat: $47.577^{\circ}$, Lon: $21.582^{\circ}, 125 \mathrm{~m}$ ASL [4]).

Figure 2 shows the modified huts for winter measurements. One goal of the winter measurements was to indicate the difference of the energy consump-

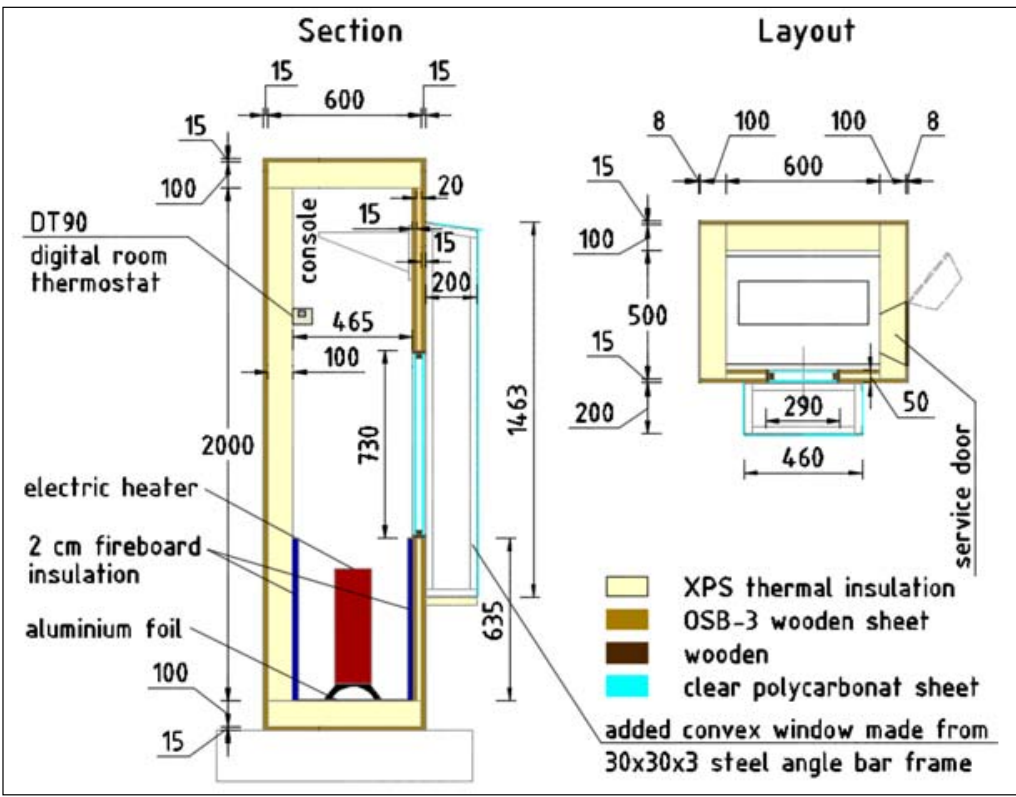

Fig. 2. Layout and section of experimental hut with added convex window for heating mode

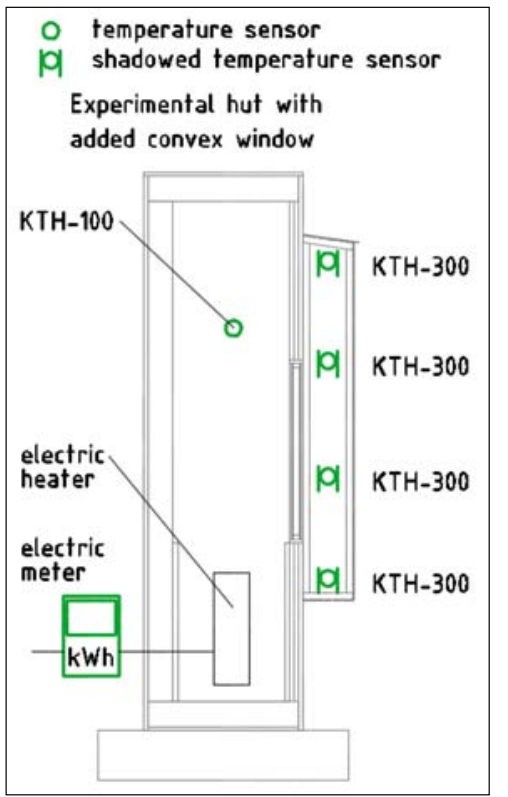

Fig. 3. Location of temperature sensors and the energy meter in the experimental hut 

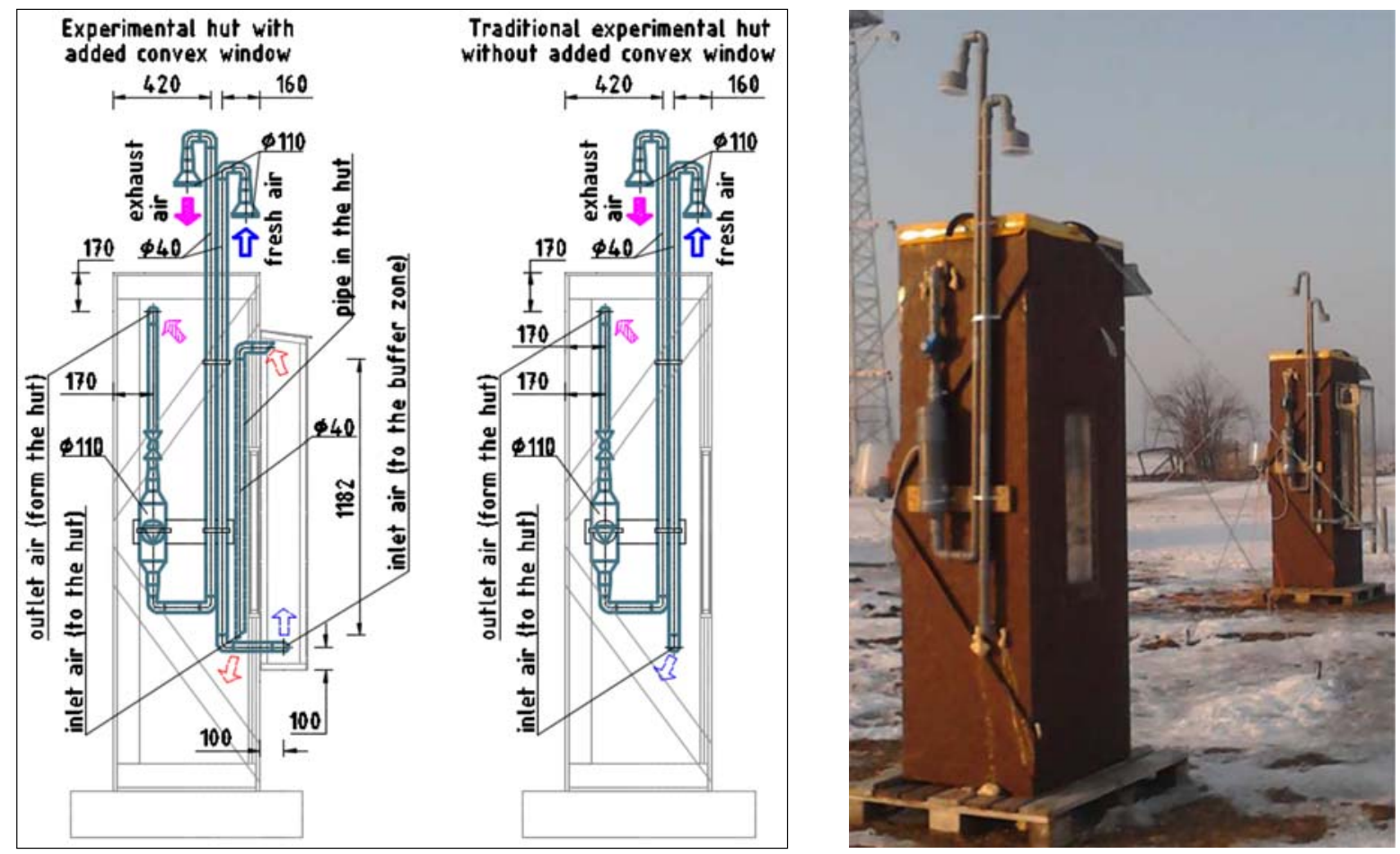

Fig. 4. The mechanical ventilation systems of the experimental huts

tions. For each hut an electrical oil filled heater was placed with $600 \mathrm{~W}$ capacity, while this was the heater with smallest capacity available on market. Because of the use of the heaters and the small sizes of the side walls fire boards were attached to the inner walls of the huts and aluminium foils were spread on the floor (Fig. 2). The temperature control was ensured by Honeywell DT90A type self-learning controller, through its application it was possible to achieve only a small temperature fluctuation of inside temperature, in spite of the difficult regulation task (large heat capacity and a controlled device with small time constant). The electrical consumption of the heaters was measured by the Home EM03 type meter, its punctuality was determined by a validated meter before the start of the measurement. The meters do not contain the data logger function, therefore daily or once in two days the results were registered manually on site.

Temperatures in huts were registered by KIMO KTH-100 and KTH-300 type of the data loggers. The situation of the sensors and meter are shown in Fig. 3. The sensors which are situated in the added convex window would be affected by solar radiation, so shading had to be installed. Shading was provided by $12 \mathrm{~cm}$ long and $30 \mathrm{~mm}$ diameter PVC shading pipes, opened on both ends. The direct contact between the sensor and the pipe was prevented by spacers made of steel wires. Measuring the temperatures in added convex window took place in four places in different heights, considering the stratification of air.
In order to show the effect of preheating measurable and identical volume flow of fresh air in both huts was needed. So mechanical system was placed in the huts, which can be seen in Fig. 4. In case of the hut with added convex window on, fresh air enters the added convex window on the side below, from there it goes to the lower part of the hut through pipe then the exhausted air leaves through the upper part of the hut. Nearly the same values of volume flow rate of the huts were achieved by the built in valve in the extract air duct of the ventilation. The income opening of the fresh air supply and the blowout of exhausted air in the hut were placed close to each other, with nearly the same external air pressures.

A VENT 100 VKO axial pipe ventilator was placed in the exhaust air duct, so that the energy balance of the hut is not affected by the heat gain of the ventilator's motor. Electrical connection of the heaters had no relations to the heater's measured connections.

The calculation of the air changes number in the huts was done by considering the changing value of the concentration of the indicator gas in the closed premise. This method gives punctual results, if the air change number is constant throughout the examination period and the indicator gas is equally spread in the closed premise. $\mathrm{CO}_{2}$ gas was used for the measurement, which was a right choice for the small volume of the huts. Filling was $6 \mathrm{~g}$ siphon cartridge as $\mathrm{CO}_{2}$ source, only few of them were used. At the beginning of the measurement data logger was placed inside the hut for $\mathrm{CO}_{2}$ concentration, and from the cartridge 
through the opened service door $\mathrm{CO}_{2}$ was injected into the hut, after that the opening was shot. For measuring the $\mathrm{CO}_{2}$ concentration TESTO 435 was used. Figure 4 shows the result of the measurement and air change number. The equation used for the calculation [5]:

$$
n=\frac{\ln \left(c_{1} / c_{2}\right)}{\tau_{1}-\tau_{2}}
$$

where

$$
\begin{aligned}
& \tau_{1}, \tau_{2}-\text { time, } \mathrm{s} \\
& c_{1}, c_{2}-\mathrm{CO}_{2} \text { concentration, } \mathrm{ppm} \\
& n \quad-\text { air change number, } \mathrm{h}^{-1}
\end{aligned}
$$

The curve must be chosen for the calculation, where after the $\mathrm{CO}_{2}$ insufflation the mixing with air has been finished and the concentration exponentially decreases. This condition was checked by using a fitted exponential regression function. Air change number of the traditional hut is: $n=2.49 \mathrm{~h}^{-1}$, as the hut with added convex window is: $n=2.69 \mathrm{~h}^{-1}$.

Throughout the winter period measurements due to the diagnostical purpose, the outside surface temperature of the hut was measured once. Moreover, infrared camera check of the huts was done. Surface temperatures were measured in sunny weather by using KIMO KTH-300. Testo 881-3 type of infrared camera was used on a cloudy day. The diagnostical examinations did not show any technical or quality problem of the huts.

Huts were modelled in Energy Plus v.8.4.0 program. The two huts were placed at a distance of 8 metres from each other in North-South direction. The height of the huts ensured the prevention of the shad- ing effect of the huts on each other as much as possible.

In the model other objects in the environment, influencing radiation conditions, were taken into account (forest, woods, buildings).

Part of meteorological parameters for the modelling have been provided by the observatory (Agrometeorological Observatory of the AKIT DTTI Agrometeorological and Agro-ecological Monitoring Centre, University of Debrecen), other part of parameters were downloaded from the professional meteorological information site www.ogimet.com. Last mentioned data are from the synoptic report from meteorological station number 12882 in Debrecen, situated $12 \mathrm{~km}$ from the observatory. Some data have been calculated. Calculation followed the method, which was described in a previous article [4] and according to the following steps:

- Horizontal infrared radiation intensity - calculated $[6,7]$ as:

where

$$
I_{\mathrm{IR}}=\varepsilon \sigma T^{4}
$$

$I_{\mathrm{IR}}-$ horizontal infrared radiation intensity, $\mathrm{W} / \mathrm{m}^{2}$,

$\varepsilon-$ sky emissivity, - ,

$\sigma$ - the Stefan-Boltzmann constant, $5.6697 \cdot 10^{-8} \mathrm{~W} / \mathrm{m}^{2} \mathrm{~K}^{4}$,

$T$ - drybulb temperature, $\mathrm{K}$.

$$
\begin{aligned}
\varepsilon & =\left[0.787+0.764 \ln \left(T_{d} / 273\right)\right] \\
& \times\left(1+0.0224 N+0.0035 N^{2}+0.00028 N^{3}\right)
\end{aligned}
$$

where

$T_{d}$ - dewpoint temperature, $\mathrm{K}$

$N$ - opaque sky cover, -

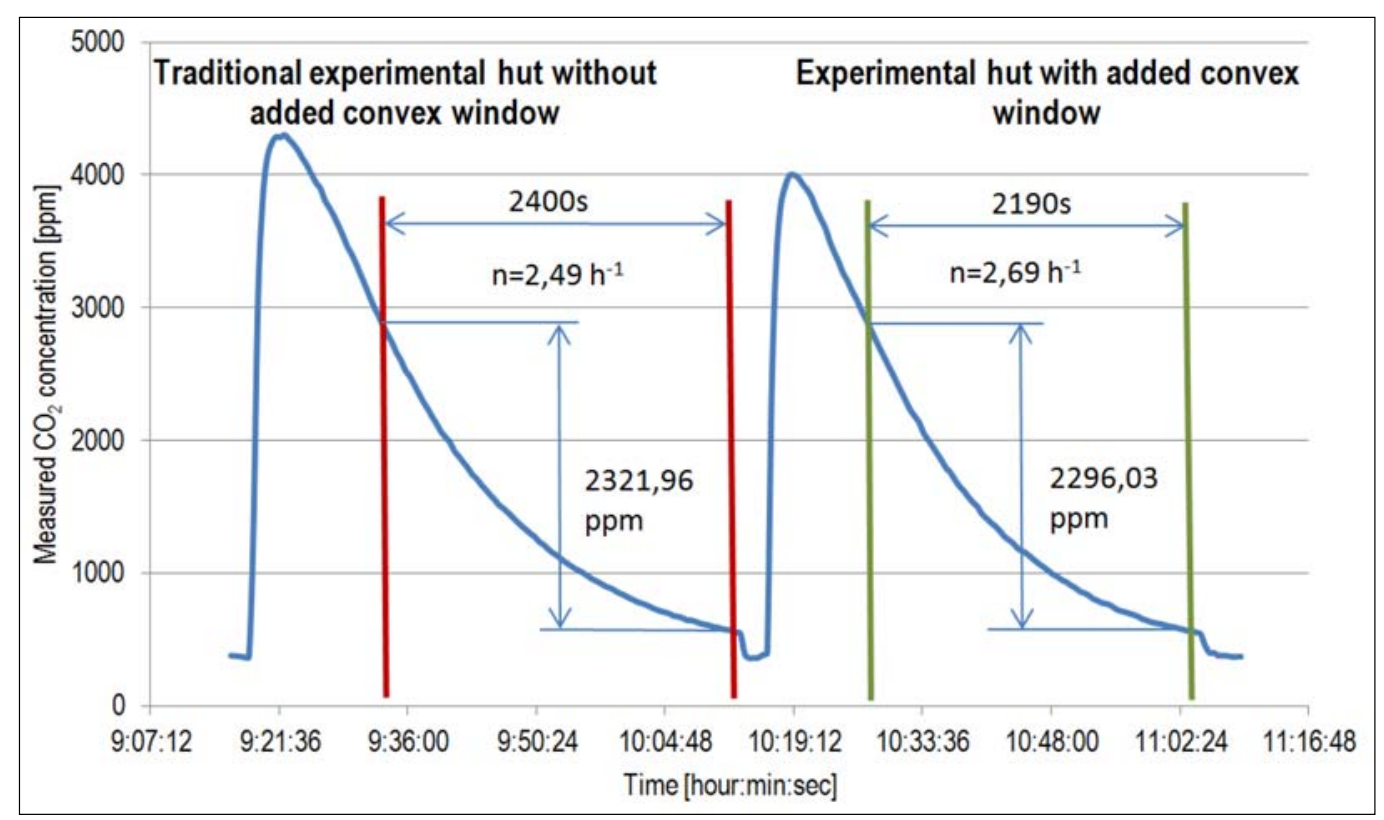

Fig. 5. Measured data of the $\mathrm{CO}_{2}$ concentrations and the air change numbers 
- Opaque sky cover - this data was missing, therefore calculation was done by taking the half of total sky cover [8].

- Snow depth - data downloaded from ogimet.com and based on notes and photos.

- Liquid precipitation depth - data from the measurements of observatory and compared with snow data.

Measurements showed that the control in the huts was able to keep the inner temperature within regulation differences. In the simulation models this difference was taken into consideration such as setpoint temperature. Certainly, the difference between the inner and the set-point temperature cannot only be caused by the difference of control, but in the period when the radiation heat gain is large, overheating can occur (against this, the automatic control system cannot do anything, as there was no cooling possibility). Due to this fact the control differences were determined based on the night hours with no overheating. Therefore the modelling of the automatic control system was not needed. Moreover, since the electrical heater's seasonal energy performance efficiency is 1 , and there are no distribution and storage losses, it is not necessary to take them into account, furthermore, losses caused by the regulation differences were compensated in simulations as mentioned above, so the re-

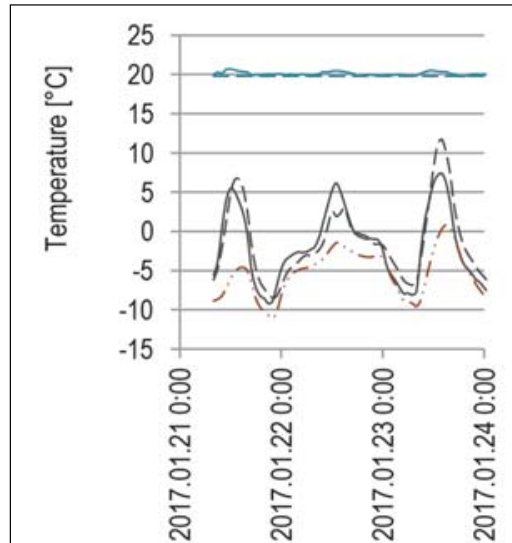

Time [year.month.day.hour:min]

a.) North - Hut with added convex window

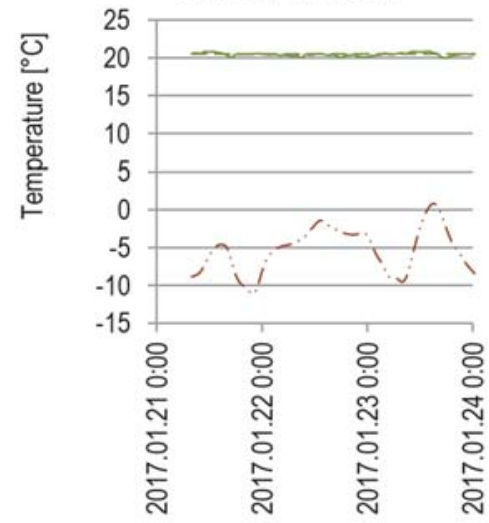

Time [year.month.day.hour:min]

d.) North - Traditional hut

(without added convex window)

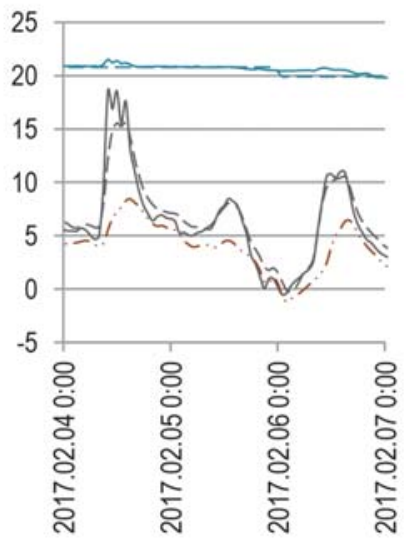

Time [year.month.day.hour:min]

b.) East - Hut with added convex window

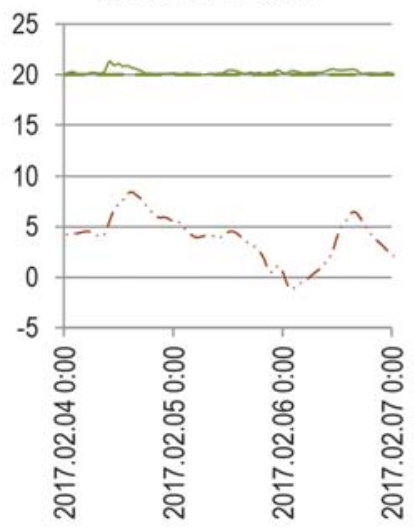

Time [year.month.day.hour:min] e.) East - Traditional hut (without added convex window)

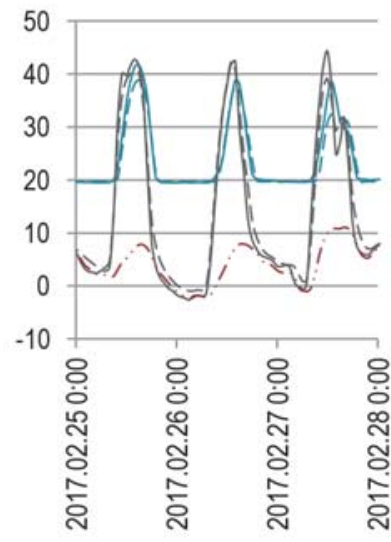

Time [year.month.day.hour:min]

c.) South - Hut with added convex window

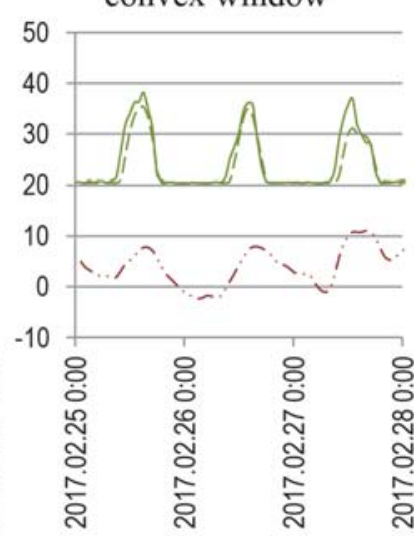

Time [year.month.day.hour:min]

f.) South - Traditional hut

(without added convex window) Legend

$$
\begin{aligned}
& \text { Measured indoor temperature of the experimental hut with added convex window } \\
& \text { Measured average temperature of the buffer zone of the added convex window } \\
& \text { Measured indoor temperature of the traditional experimental hut without added convex window } \\
& \text { Simulation result of the indoor temperature of the experimental hut with added convex window } \\
& ---\quad \text { Simulation result of the average temperature of the buffer zone of the added convex window } \\
& ---- \\
& \text { Simulation result of the indoor temperature of the traditional experimental hut without add. conv. wind. } \\
& \text { Measured outdoor temperature }
\end{aligned}
$$

Fig. 6. Results of temperatures from simulations and measured data (Time: Central European Time, CET) 


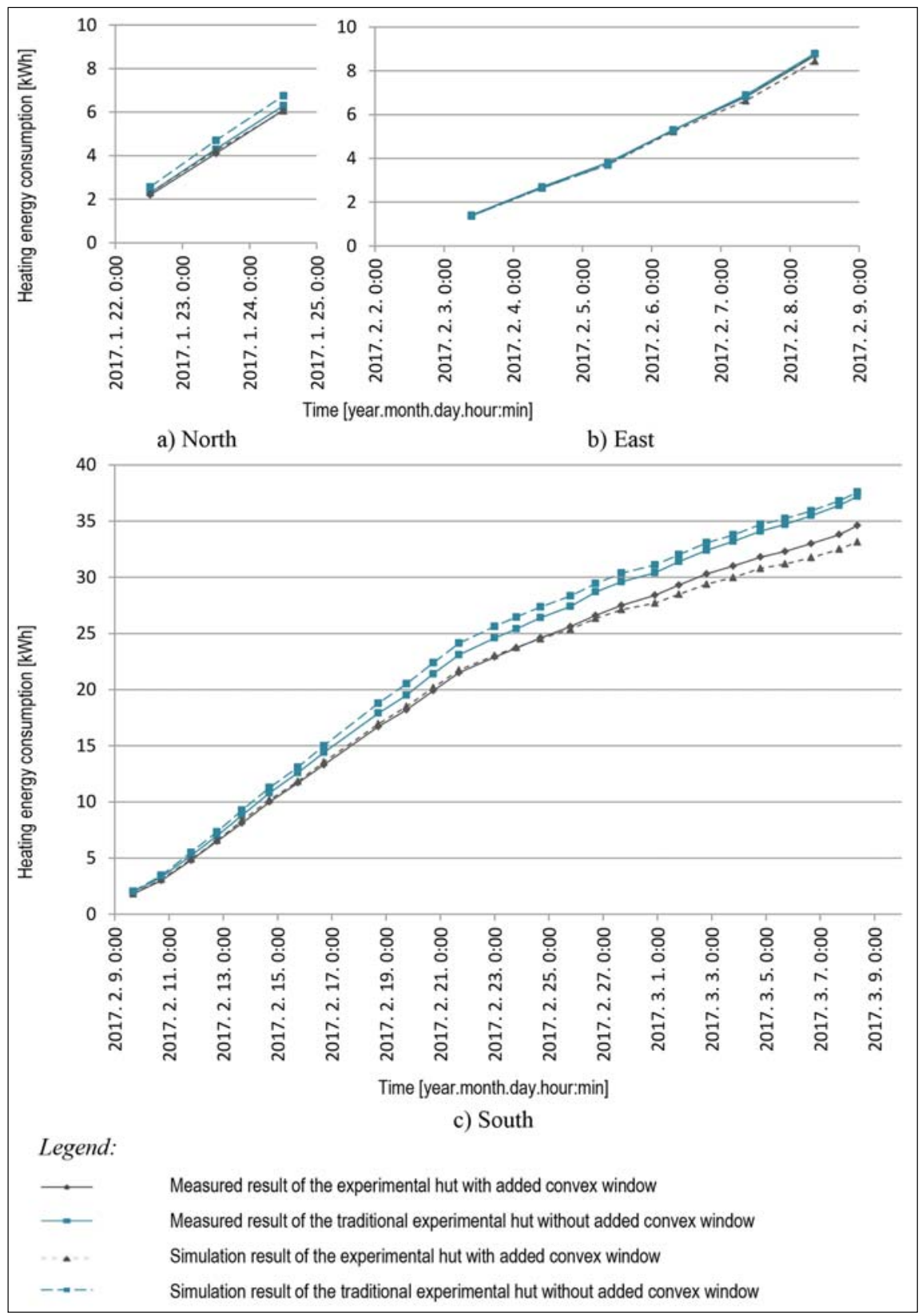

Fig. 7. Results of heating energy consumptions from simulations and measured data (Time: Central European Time, CET)

sults of energy need for heating from the simulated huts mean the heating energy consumption as well.

\section{Results}

Figures 6 and 7 show the calculated and measured temperatures and the calculated and measured energy consumption, respectively.

\section{Discussion}

Based on the measurements it can be seen that the average temperature of the buffer zone is higher during the day than the outside air temperature. This of course decreases the ventilation loss of the hut with added convex window, the transmission loss of the original window and the wall area covered with added convex window. Heating energy consumption of the hut with added convex window is lower, and when the indoor temperature exceeds the set point temperature the value of overheating is higher, which confirms the previous statement. These are in accordance with the simulation's results.

\section{Conclusion}

It was proven by dynamic simulations supported by the measured data of the experimental huts that pre- 
heating of fresh air in the added convex window results in considerable decrease of ventilation heat loss, this way increasing the renewable share in covering the heating energy need.

\section{Acknowledgements}

The work/publication is supported by the EFOP-3.6.116-2016-00022 project. The project is co-financed by the European Union and the European Social Fund.

\section{References}

[3] Kerekes A. (2017), Impact of added convex windows on energy need for heating in different climatic zones. In: Design to Thrive. PLEA 2017 Conference, Luisa Brotas, Susan Roaf, Fergus Nicol (eds), PLEA, Edinburgh, pp. 628-634.

[4] Kerekes A. (2017), Ablakcseres vs. előtétablak. Magyar Épületgépészet, LXVI(2017/4), 8-12.
[5] Kerekes A., Zöld A. (2016), Added convex windows for improving the energy balance. Applied Mechanics and Materials, 824, 339-346.

[6] Kerekes A., et al. (2018), Prevention of summer overheating of buildings with added convex windows. International Review of Applied Sciences and Engineering, 9(1), 41-48.

[7] Fekete I., et al. (1985), Épületfizika kézikönyv, Müszaki Könyvkiadó Bp. 389 p.

[8] Walton G. N. (1985), Thermal Analysis Research Program - Reference Manual, NBSIR 83-2655, U.S. Department of Commerce, March (1983), Update 1985.

[9] Clark G., Allen C. (1978), The Estimation of Atmospheric Radiation for Clear and Cloudy Skies. In: Proceedings 2nd National Passive Solar Conference (AS/ISES), pp. 675-678.

[10] Jentsch M. F., et al. (2008), Climate change future proofing of buildings - Generation and assessment of building simulation weather files. Energy and Buildings, 40, 21482168 . 